\title{
THE CAUCHY-HEAVISIDE EXPANSION FORMULA AND THE BOLTZMANN-HOPKINSON PRINCIPLE OF SUPERPOSITION*
}

\author{
BY F. D. MURNAGHAN
}

1. Introduction. It is the object of the present note to point out that the well known expansion theorem of Heaviside is an immediate corollary of the classical method given by Cauchy for finding a particular solution of a linear, nonhomogeneous, ordinary differential equation when the general solution of the corresponding homogeneous equation is known. Various proofs of Heaviside's theorem have been given by Bromwich and Wagner, using contour integrals, by Carson and others and it seems hardly possible that the direct connection with Cauchy's method can have escaped attention. This method is interesting, furthermore, since it furnishes a rigorous proof of the useful Boltzmann-Hopkinson principle of superposition. $\dagger$ The explicit form of the expansion theorem in the case when the characteristic equation has multiple roots is also given.

2. Cauchy's Method. Although Cauchy's method is classical and to be found in the older books, it does not seem to find a place in the more modern texts and it will, therefore, be convenient to give an indication of its derivation. Let us suppose

* Presented to the Society, under a different title, September 9, 1926.

$\dagger$ The following citations may serve to give a proper orientation: Bromwich, T. J., Normal coordinates in dynamical systems, Proceedings of the London Society, vol. 15 (1916), pp. 401-448. Wagner, K. W.,Über eine Formel von Heaviside zur Berechnung von Einschaltvorgängen, Archiv für Elektrotechnik, vol. 4 (1916), pp. 159-193. Carson, J. R., On a general expansion theorem for the transient oscillations of a connected system, Physical Review, vol. 10 (1917), pp. 217-225. Carson, J. R., 1 heory of the transient oscillations of electrical networks and transmission lines, Transactions A. I. E. E., vol. 38 (1919), pp. 345-427. 
that we wish to find a particular solution of the linear differential equation of the $n$th order

$$
\phi(D) u=f(t) ; \quad \phi(D) \equiv a_{0} D^{n}+\cdots+a_{n},
$$

where $u$ is the dependent variable, $t$ the independent variable and $D \equiv d / d t$. The coefficients $a$ are either constants or functions of $t$. If $\left(v_{1}, \cdots, v_{n}\right)$ are distinct solutions of the corresponding homogeneous equation

$$
\phi(D) v=0,
$$

its general solution is

$$
v=C_{1} v_{1}+\cdots+C_{n} v_{n},
$$

where the $C$ 's are arbitrary constants. We choose these so that $v$ and its derivatives with respect to $t$ up to the $(n-2) \mathrm{d}$ vanish, when $t=\tau$ the $(n-1)$ st derivative taking the value $f(\tau) / a_{0}(\tau) ; \tau$ being a fixed but arbitrary value of $t$. To find the proper values for the $C$ 's, we have merely to solve a system of $n$ linear equations whose determinant is the Wronskian $\left|v_{1} v_{2}^{\prime} \cdots v_{n}^{(n-1)}\right|$ and this does not vanish on account of the distinctness of the $v$ 's. When the values of the $C$ 's as thus determined are entered in (2), $v$ becomes a function of $t$ and $\tau$, and to stress this we may write it as $v(t, \tau)$. Then Cauchy's contention is that the integral

$$
u(t)=\int_{0}^{t} v(t, \tau) d \tau
$$

is a particular solution of (1). We have, in fact, from (3)

$$
D u=\int_{0}^{t} D v(t, \tau) d \tau+v(t, t)=\int_{0}^{t} D v(t, \tau) d \tau,
$$

since $v(\tau, \tau)$ vanishes by definition for every value of $\tau$. Similarly

while

$$
D^{2} u=\int_{0}^{t} D^{2} v(t, \tau) d \tau, \cdots, D^{n-1} u=\int_{0}^{t} D^{n-1} v(t, \tau) d \tau,
$$

$D^{n} u=\int_{0}^{t} D^{n_{v}}(t, \tau) d \tau+\left\{D^{n-1} v(t, \tau)\right\}_{\tau=t}=\int_{0}^{t} D^{n_{v}}(t, \tau) d \tau+\frac{f(t)}{a_{0}(t)}$, 
so that

$$
\phi(D) u=\int_{0}^{t} \phi(D) v(t, \tau) d \tau+f(t)=f(t)
$$

The particular solution $u$ of (1) obtained in this way is characterized by the fact that it vanishes with its derivatives up to the $(n-1)$ st when $t=0$; the $n$th derivative having, consequently, the value $f(0) / a_{0}(0)$ when $t=0$. It is apparent from the definition of the $C$ 's occurring in (2) that they may be obtained from the values they would have if $f(\tau)$ were $\equiv 1$ by multiplication by $f(\tau)$. Hence if we denote by $\psi(t, \tau)$ the particular function $v(t, \tau)$ corresponding to the particular problem where $f(t) \equiv 1$ we have $v(t, \tau) \equiv f(t) \psi(t, \tau)$, and (3) may be written in the form

$$
u(t)=\int_{0}^{t} f(\tau) \psi(t, \tau) d \tau .
$$

Denoting by $\xi(t, 0)$ the particular solution corresponding to $f(t) \equiv 1$ we have

$$
\xi(t, 0) \equiv \int_{0}^{t} \psi(t, \tau) d \tau
$$

which suggests the notation

$$
\xi(t, \tau) \equiv \int_{\tau}^{t} \psi(t, s) d s
$$

with the relation

$$
\frac{\partial \xi(t, \tau)}{\partial \tau}=-\psi(t, \tau) .
$$

An integration of ( $\left.3^{\text {bis }}\right)$ by parts then yields

$$
\left\{\begin{aligned}
u(t) & =[-f(t) \xi(t, t)+f(0) \xi(t, 0)]+\int_{0}^{t} \xi(t, \tau) \frac{d f}{d \tau} d \tau \\
& =f(0) \xi(t, 0)+\int_{0}^{t} \xi(t, \tau) \frac{d f}{d \tau} d \tau, \text { since } \xi(t, t)=0 .
\end{aligned}\right.
$$

This is the mathematical statement of the Boltzmann-Hopkinson principle of superposition according to which we are able 
to build up from a particular solution $\xi(t, \tau)$ of the differential equation (1) when $f(t) \equiv 1$, a particular solution of this equation when $f(t)$ is an arbitrary function of $t$. The particular solution obtained in this way is characterized by the fact that it vanishes, together with its derivatives with respect to $t$ up to the $(n-1)$ st, when $t=0$.

3. The Particular Case when the Coefficients of $\phi(D)$ are Constant. In the first case we shall consider the general case where the zeros of the polynomial $\phi(D)$ are all distinct and we shall write

$$
\phi(D) \equiv a_{0}\left(D-r_{1}\right)\left(D-r_{2}\right) \cdots\left(D-r_{n}\right) .
$$

We may choose as our $n$ distinct solutions of $\left(1^{\prime}\right)$ the functions

$$
v_{1}=e^{r_{1}(t-r)}, \cdots, v_{n}=e^{r_{n}(t-r)},
$$

and our equations to determine the constants $C$ of (2) are

$$
\left\{\begin{array}{c}
C_{1}+\cdots+C_{n}=0 \\
r_{1} C_{1}+\cdots+r_{n} C_{n}=0 \\
\cdots \cdot \cdots \cdot \cdot \cdot \\
r_{1}{ }^{n-1} C_{1}+\cdots+r_{n}^{n-1} C_{n}=\frac{f(\tau)}{a_{0}}
\end{array}\right.
$$

These equations show that $\left(C_{1}, \cdots, C_{n}\right)$ are the coefficients of the analysis of $f(\tau) / \phi(D)$ into its simple fractions. In fact if we write

$$
\frac{f(\tau)}{\phi(D)} \equiv \frac{C_{1}}{D-r_{1}}+\cdots+\frac{C_{n}}{D-r_{n}},
$$

and expand each of the fractions on the right-hand side near $D=\infty$, we find on observing that $D=\infty$ is a zero of order $n$ of the left-hand side, the first term in its expansion being $f(\tau) /\left(a_{0} D^{n}\right)$, exactly the equations (6) to determine the $C$ 's. Further more on observing that $D=r_{p}$ is a simple pole with residue $f(\tau) / \phi^{\prime}\left(r_{p}\right)$ of the left-hand side we obtain the relations

$$
C_{p}=\frac{f(\tau)}{\phi^{\prime}\left(r_{p}\right)}, \quad(p=1, \cdots, n) .
$$


Hence the function $v(t, \tau)$ is

so that

$$
v(t, \tau) \equiv f(\tau) \sum_{p=1}^{n} \frac{e^{r_{p}(t-\tau)}}{\phi^{\prime}\left(r_{p}\right)}
$$

and

$$
\psi(t, \tau) \equiv \sum_{p=1}^{n} \frac{e^{r_{p}(t-\tau)}}{\phi^{\prime}\left(r_{p}\right)}
$$

The relation

$$
\xi(t, \tau) \equiv \sum_{p=1}^{n} \frac{e^{r_{p}(t-r)}}{r_{p} \phi^{\prime}\left(r_{p}\right)}-\sum_{1}^{n} \frac{1}{r_{p} \phi^{\prime}\left(r_{p}\right)} .
$$

$$
\frac{1}{\phi(D)} \equiv \sum_{p=1}^{n} \frac{1}{\phi^{\prime}\left(r_{p}\right)\left(D-r_{p}\right)}
$$

obtained above shows that

so that we may write

$$
\frac{1}{\phi(0)}=-\sum_{p=1}^{n} \frac{1}{r_{p} \phi^{\prime}\left(r_{p}\right)},
$$

$$
\xi(t, \tau)=\frac{1}{\phi(0)}+\sum_{p=1}^{n} \frac{e^{r_{p}(t-\tau)}}{r_{p} \phi^{\prime}\left(r_{p}\right)},
$$

and, in particular,

$$
\xi(t) \equiv \xi(t, 0)=\frac{1}{\phi(0)}+\sum_{p=1}^{n} \frac{e^{r_{p} t}}{r_{p} \phi^{\prime}\left(r_{p}\right)} .
$$

It is this particular form of Cauchy's result which yields immediately the Heaviside expansion theorem. Before proceeding to show this it will be well, however, to see what modification is introduced when two or more of the zeros of $\phi(D)$ coincide. For simplicity of presentation let us suppose that $r_{2}=r_{1}$, the other roots being all different from $r_{1}$ and from one another. Then we take

$$
v_{1}=e^{r_{1}(t-r)}, v_{2}=(t-\tau) e^{r_{1}(t-\tau)}, v_{3}=e^{r_{3}(t-\tau)}, \cdots, v_{n}=e^{r_{n}(t-r)} \text {, }
$$

and the equations to determine the constants $C$ are

$$
\begin{gathered}
C_{1}+0 \cdot C_{2}+C_{3}+\cdots+C_{n}=0, \\
r_{1} C_{1}+1 C_{2}+r_{3} C_{3}+\cdots+r_{n} C_{n}=0, \\
r_{1}^{2} C_{1}+2 r_{1} C_{2}+r_{3}^{2} C_{3}+\cdots+r_{n}^{2} C_{n}=0, \\
\cdot \cdot \cdot \cdot \cdot \cdot \cdot \cdot \cdot \cdot \cdot \cdot \cdot \cdot \cdot \cdot \cdot \cdot \cdot r_{n}^{n-1} C_{n}=\frac{f(r)}{a_{0}},
\end{gathered}
$$


the coefficient of $C_{2}$ in each equation being the derivative with respect to $r_{1}$ of the coefficient of $C_{1}$. These equations show, just as before, that $\left(C_{1}, \cdots, C_{n}\right)$ are the coefficients in the analysis of $f(\tau) / \phi(D)$ into its simple fractions; i.e.,

$$
\frac{f(\tau)}{\phi(D)} \equiv \frac{C_{1}}{D-r_{1}}+\frac{C_{2}}{\left(D-r_{1}\right)^{2}}+\frac{C_{3}}{D-r_{3}}+\cdots+\frac{C_{n}}{D-r_{n}} .
$$

Developing this identity in the neighborhood of the double zero $D=r_{1}$, and setting

we have

$$
\frac{\left(D-r_{1}\right)^{2}}{\phi(D)} \equiv F(D)
$$

$$
C_{1}=f(\tau) F^{\prime}\left(r_{1}\right), \quad C_{2}=f(\tau) F\left(r_{1}\right) .
$$

The part of the function $\xi(t)$ corresponding to the double zero $r=r_{1}$ may be conveniently expressed as follows. Writing $C_{p}=f(\tau) A_{p},(p=1, \cdots, n)$, so that

$$
\frac{1}{\phi(D)}=\frac{A_{1}}{D-r_{1}}+\frac{A_{2}}{\left(D-r_{1}\right)^{2}}+\cdots+\frac{A_{n}}{D-r_{n}},
$$

we have, for the part of $\xi(t)$ in question, to evaluate the integral

$$
\int_{0}^{t}\left\{A_{1}+A_{2}(t-\tau)\right\} e^{r_{1}(t-\tau)} d \tau .
$$

If we write $t-\tau=s$, this becomes

$$
\int_{0}^{t}\left(A_{1}+A_{2} s\right) e^{r_{1} 8} d s=\frac{A_{2}}{r_{1}} t e^{r_{1} t}+\left(\frac{A_{1}}{r_{1}}-\frac{A_{2}}{r_{1}^{2}}\right) e^{r_{1} t}-\left(\frac{A_{1}}{r_{1}}-\frac{A_{2}}{r_{1}^{2}}\right) ;
$$

as $A_{1}=F^{\prime}\left(r_{1}\right)$ and $A_{2}=F\left(r_{1}\right)$, the variable part of this may be written in the form

$$
\frac{F\left(r_{1}\right)}{r_{1}} t e^{r_{1} t}+\left\{\frac{d}{d r_{1}}\left(\frac{F\left(r_{1}\right)}{r_{1}}\right)\right\} e^{r_{1} t} .
$$

In an exactly similar manner it can be shown that the variable part of $\xi(t)$ corresponding to a multiple root of order $m$ is 


$$
\begin{array}{r}
\frac{1}{(m-1) !}\left[\left(\frac{F\left(r_{1}\right)}{r_{1}}\right) t^{m-1} e^{r_{1} t}+(m-1)\left\{\frac{d}{d r_{1}}\left(\frac{F\left(r_{1}\right)}{r_{1}}\right)\right\} t^{m-2} e^{r_{1} t}\right. \\
\left.+\cdots+\left\{\frac{d^{m-1}}{d r_{1}{ }^{m-1}}\left(\frac{F\left(r_{1}\right)}{r_{1}}\right)\right\} e^{r_{1} t}\right]
\end{array}
$$

where $F(D) \equiv\left(D-r_{1}\right)^{m} / \phi(D)$; the expression in parenthesis being written with binomial coefficients. Thus in the case of a triple root the constants $C$ are given by the equation

$$
\frac{f(\tau)}{\phi(D)} \equiv \frac{C_{1}}{D-r_{1}}+\frac{C_{2}}{\left(D-r_{1}\right)^{2}}+\frac{2 ! C_{3}}{\left(D-r_{1}\right)^{3}}+\cdots
$$

It follows from (10), on putting $D=0$, that the constant part of $\xi(t)$ is $1 / \phi(0)$. These results furnish the general expression for the particular solution of $\phi(D) u=1$ which vanishes with its derivatives up to the $(n-1)$ st when $t=0$.

4. The Heaviside Problem. In his study of electric oscillations Heaviside had to consider a system of ordinary linear differential equations of the type

$$
\sum_{\alpha=1}^{k} a_{r \alpha} x_{\alpha}=E_{r}(t), \quad(r=1, \cdots, k) .
$$

Here the $a$ 's are polynomials in the operator $D$ whose coefficients may involve the independent variable $t$ (although Heaviside was particularly interested in the case where these coefficients are constants) and expressions for the $k$ dependent variables are sought for, the functions $E(t)$ on the right-hand sides of the equations (11) being given. All the dependent variables but one, $x_{s}$ say, may be eliminated by operating on the equations (11) with the cofactors of the sth column of the determinant $\left|a_{r s}\right| \equiv \phi(D)$. Denoting these cofactors by $A_{18}$, $\cdots, A_{k s}$ we find

$$
\phi(D) x_{s}=\sum_{r=1}^{k} A_{r s} E_{r}(t) .
$$

Let us now confine our attention to the case where $E_{p}(t)=1$, all other $E$ 's being zero, and where, in addition, the coefficients 
of the polynomials $a_{r s}$ are constants. In this event, the operators $\phi(D)$ and $A_{p s}$ are commutative and we first set up that particular solution of

$$
\phi(D) y_{p}=1
$$

which vanishes, together with its derivatives up to that of the $(n-1)$ st order, when $t=0, n$ being the degree of the polynomial $\phi(D)$. Then $x_{s p}=A_{p s} y_{p}$ is a particular solution of the equation $\phi(D) x_{s}=A_{p_{s}}$ and it is characterized by the fact that its derivatives up to an order one less than the degree in $D$ of the elements $a_{r s}$ vanish when $t=0$. From the exponential form already given for $y_{p}(t, 0)$ in the case where the zeros of $\phi(D)$ are simple, it follows that

$$
x_{s p}(t, 0)=A_{p_{s}} y_{p}(t, 0)=\sum_{\alpha=1}^{n} \frac{A_{p s}\left(r_{\alpha}\right) e^{r_{\alpha}(t)}}{r_{\alpha} \phi^{\prime}\left(r_{\alpha}\right)}+\frac{A_{p s}(0)}{\phi(0)},
$$

which is Heaviside's expansion formula. If now we consider the equation $\phi(D) u_{p}=E_{p}(t)$, a particular solution is

$$
u_{p}(t)=E_{p}(0) y_{p}(t, 0)+\int_{0}^{t} \frac{d E_{p}}{d \tau} y_{\tilde{r}}(t, \tau) d \tau,
$$

this particular solution being characterized by the fact that it, together with all its derivatives up to the $(n-1)$ st, vanish when $t=0$. The function

$$
\bar{x}_{s p}=A_{p s} u_{p}(t)=E_{p}(0) x_{s p}(t, 0)+\int_{0}^{t} \frac{d E_{p}}{d \tau} x_{s p}(t, \tau) d \tau
$$

is, accordingly, a particular solution of the equation

$$
\phi(D) \bar{x}_{s p}=A_{p s} E_{p}(t) .
$$

Adding with respect to $p$ from 1 to $k$, we find that the set of functions

$$
x_{\iota}=\sum_{p=1}^{k}\left\{E_{p}(0) x_{s p}(t, 0)+\int_{0}^{t} \frac{d E_{p}}{d \tau} x_{s p}(t, \tau) d \tau\right\}
$$

furnish that particular solution of the equations(11) which is 
characterized by the fact that all the $x$ 's and their derivatives up to an order one less than the degree of the $a_{r s}$ in $D$ vanish when $t=0$.

When the zeros of $\phi(D)$ are not distinct we avail ourselves of the relations

$$
\begin{aligned}
D\left(t e^{r t}\right) & =r t e^{r t}+e^{r t}, \quad D\left(t^{m} e^{r t}\right)=r t^{m} e^{r t}+m t^{m-1} e^{r t}, \\
D^{2}\left(t^{m} e^{r t}\right) & =r^{2} t^{m} e^{r t}+2 r m t^{m-1} e^{r t}+m(m-1) t^{m-2} e^{r t}, \\
D^{3}\left(t^{m} e^{r t}\right) & =r^{3} t^{m} e^{r t}+3 r^{2} m t^{m-1} e^{r t}+3 r m(m-1) t^{m-2} e^{r t} \\
& +m(m-1)(m-2) t^{m-3} e^{r t},
\end{aligned}
$$

which show that

$$
\begin{aligned}
A_{p s}\left(t^{m} e^{r t}\right)=A_{p s}(r) & t^{m} e^{r t}+A_{p s}^{\prime}(r) m t^{m-1} e^{r t} \\
& +A_{p_{s}^{\prime \prime}}^{\prime \prime}(r) \frac{m(m-1)}{1 \cdot 2} t^{m-2} e^{r t}+\cdots
\end{aligned}
$$

When, then, we are operating with $A_{p s}$ on a term such as

$$
\frac{1}{(m-1) !}\left\{\frac{F\left(r_{1}\right)}{r_{1}}-t^{m-1} e^{r_{1} t}+(m-1) \frac{d}{d r_{1}} \frac{F\left(r_{1}\right)}{r_{1}} t^{m-2} e^{r_{1} t}+\cdots\right\}
$$

which corresponds to a multiple root $r_{1}$ of order $m$, we obtain

$$
\begin{aligned}
& \frac{1}{(m-1) !}\left\{\frac{A_{p s}\left(r_{1}\right) F\left(r_{1}\right)}{r_{1}}-t^{m-1}+(m-1) \frac{d}{d r_{1}}\left(\frac{A_{p s}\left(r_{1}\right) F\left(r_{1}\right)}{r_{1}}\right) t^{m-2}\right. \\
& \left.+\frac{(m-1)(m-2)}{1 \cdot 2} \frac{d^{2}}{d r_{1}^{2}}\left(\frac{A_{p s}\left(r_{1}\right) F\left(r_{1}\right)}{r_{1}}\right) t^{m-3}+\cdots\right\} e^{r_{1} t} ;
\end{aligned}
$$

and these terms must be used in the expansion formula (12). Johns Hopkins University 